MATHEMATICS OF COMPUTATION

Volume 72, Number 243, Pages 1099-1115

S 0025-5718(02)01467-9

Article electronically published on December 3, 2002

\title{
FINITE ELEMENT APPROXIMATION OF SPECTRAL PROBLEMS WITH NEUMANN BOUNDARY CONDITIONS ON CURVED DOMAINS
}

\author{
ERWIN HERNÁNDEZ AND RODOLFO RODRÍGUEZ
}

\begin{abstract}
This paper deals with the finite element approximation of the spectral problem for the Laplace equation with Neumann boundary conditions on a curved nonconvex domain $\Omega$. Convergence and optimal order error estimates are proved for standard piecewise linear continuous elements on a discrete polygonal domain $\Omega_{h} \not \subset \Omega$ in the framework of the abstract spectral approximation theory.
\end{abstract}

\section{INTRODUCTION}

In this paper we analyze an elementary problem: the piecewise linear finite element approximation of eigenvalues and eigenfunctions of the Laplace operator with a Neumann boundary condition on a curved domain $\Omega$.

Since the Neumann condition is a natural condition, in principle, a conforming finite element space could theoretically be defined on a curved triangulation fitting exactly the boundary of $\Omega$ (see, for instance, Chapter 8 of [5]). Then, to effectively solve the problem, the integrals for the entries of the stiffness and mass matrices on the curved triangles should be computed by means of a quadrature rule. Thus, the method turns out to be actually nonconforming.

However, for piecewise linear elements, the most common procedure in practice is to consider a mesh $\mathcal{T}_{h}$ formed by straight triangles. Then, the integrals of the entries of both matrices are actually computed on a discrete domain $\Omega_{h}:=\bigcup\left\{T \in \mathcal{T}_{h}\right\}$, which do not coincide with $\Omega$, thus yielding also a nonconforming method. As we show in this paper, this coarser procedure leads anyway to optimal order approximation results.

When $\Omega_{h} \subset \Omega$, the standard theory applies almost directly. In fact, for Dirichlet boundary conditions the method turns out to be conforming since the discrete space is a subset of $\mathrm{H}_{0}^{1}(\Omega)$ (see, e.g., the book by Raviart and Thomas [16]). For Neumann boundary conditions the method is not conforming, but convergence and optimal error estimates can be proved as rather straightforward consequences of the standard Strang's lemma, estimates like Lemma 5.2-5.3 in Raviart and Thomas's

Received by the editor February 2, 2001 and, in revised form, September 28, 2001.

2000 Mathematics Subject Classification. Primary 65N25, 65N30; Secondary 70J30.

Key words and phrases. Finite element spectral approximation, curved domains.

The first author was supported by FONDECYT 2000114 (Chile). The second author was partially supported by FONDECYT 1990346 and FONDAP in Applied Mathematics (Chile). 
book [16], and the classical abstract spectral approximation theory by Osborn [15] (see also the monograph by Babuška and Osborn [1]).

The situation is quite different when $\Omega_{h} \not \subset \Omega$. The finite element approximation of the linear boundary value problem associated to the Laplace operator has been extensively studied. Different approaches have been considered, for instance by Ciarlet and Raviart 8], Zlámal [23, and Scott [17. (see also [18, 2], and [22]).

However, these results do not lead to a similar analysis for the corresponding eigenvalue problem. Indeed, convergence results for a linear boundary value problem do not necessarily imply similar results for its associated spectral one. This is particularly true for nonconforming and mixed methods (see, for instance, 3 , 4 for examples of methods working out for the linear boundary value problem but failing for the associated eigenvalue problem).

In fact, to apply the spectral approximation theory stated in [1, one needs convergence in $\mathrm{L}^{2}$ norm for the involved discrete operators, as well as optimal order $\mathrm{L}^{2}$ and $\mathrm{H}^{1}$ convergence results for the associated linear boundary value problem with homogeneous boundary conditions. For instance, for the Dirichlet problem on a curved domain with a smooth boundary, such results have been only recently proved by Bramble and King in Proposition 1 of [6], where the transfer of nonhomogeneous boundary data is analyzed.

For the eigenvalue problem, the first proof of convergence valid in the case $\Omega_{h} \not \subset$ $\Omega$ was given by Vanmaele and Ženíšek [19] just a few years ago. They prove it for simple eigenvalues and Dirichlet boundary conditions by using the min-max characterization. These results have been further extended by the same authors to include multiple eigenvalues [20] and numerical integration effects 21] (thus allowing us to considering nonconstant coefficients).

Around the same time, Lebaud analyzed in [14] a similar problem also for simple eigenvalues and Dirichlet boundary conditions. She allowed for nonconstant coefficients in the differential operator, but assumed exact integration. She used the spectral approximation theory stated in [1] and optimal convergence results like those in 6. She proved that, when isoparametric elements of degree $k$ are used and the eigenfunctions are sufficiently smooth, the approximation of the eigenvalues is of order $\mathcal{O}\left(h^{2 k}\right)$ instead of $\mathcal{O}\left(h^{k+1}\right)$.

In the case of Neumann boundary conditions, to the best of the authors' knowledge, no approximation result has been proved yet for the eigenvalue problem. Moreover, it is not apparent that the theory in [19] nor that in [14] could be extended to provide optimal error estimates in this case. In fact, both theories rely on estimates like (4.8) in [19], which are valid for functions vanishing on $\partial \Omega_{h}$ or on $\partial \Omega$ like the eigenfunctions of a Dirichlet problem, but not for those of a Neumann one.

For instance, for piecewise linear elements, the techniques in 14 clearly do not apply to the Neumann problem. To see this, notice that these results depend on the estimate (4.4) of that paper:

$$
\left\|\left(T-T_{h}\right) u\right\|_{0,2, \mathbb{R}^{2}}=\mathcal{O}\left(h^{2}\right),
$$

where $T u$ is the solution of the Laplace equations on $\Omega$ with right-hand side $u$, and $T_{h} u$ is its piecewise linear finite element approximation on $\Omega_{h}$, obtained by using as its right-hand side the extension of $u$ by zero outside of $\Omega\left(T u\right.$ and $T_{h} u$ are also extended by zero outside of their domains). Estimate (1.1) is proved in [14] as a 
consequence of the following three inequalities:

$$
\begin{aligned}
\left\|\left(T-T_{h}\right) u\right\|_{m, 2, \Omega \cap \Omega_{h}} & \leq C h^{2-m}\|T u\|_{2,2, \Omega}, \quad \text { for } m=0,1, \\
\|T u\|_{0,2, \Omega \backslash\left(\Omega \cap \Omega_{h}\right)} & \leq C h^{2}\|T u\|_{2,2, \Omega}, \\
\left\|T_{h} u\right\|_{0,2, \Omega_{h} \backslash\left(\Omega \cap \Omega_{h}\right)} & \leq C h^{2}\left\|T_{h} u\right\|_{1,2, \Omega \cap \Omega_{h}} .
\end{aligned}
$$

To the best of the authors' knowledge estimate (1.2) has not been proved for $m=0$ and Neumann boundary conditions. Furthermore, estimate (1.4) is clearly not valid in this case.

This shows the necessity of considering a more subtle extension of the data $u$ outside of $\Omega$. One such extension, which preserves the eigenvalues of the Neumann problem, is introduced and analyzed in the next section.

The goal of this paper is to analyze the piecewise linear finite element approximation of the spectral problem for the Laplace equation with Neumann boundary conditions on curved nonconvex domains. We prove optimal order $\mathrm{L}^{2}$ and $\mathrm{H}^{1}$ error estimates for the eigenfunctions, and a double order for the eigenvalues. These estimates are proved to be valid for any piecewise smooth Lipschitz domain, for which the eigenfunctions do not necessarily belong to $\mathrm{H}^{2}(\Omega)$.

The outline of the paper is as follows. In Section 2 we present the model problem, the meshes to be used, the finite element approximation, and some basic results. Then, in Section 3, we prove optimal order error estimates for the approximate eigenfunctions and eigenvalues in the framework of the spectral approximation theory stated in [1].

\section{Statement of the Problem}

Let $\Omega \subset \mathbb{R}^{2}$ be a bounded open domain, in general nonconvex, with a piecewise smooth (e.g., $\mathcal{C}^{2}$ ) Lipschitz boundary $\Gamma:=\partial \Omega$. Consider the eigenvalue problem for the shifted Laplace operator in $\Omega$ with Neumann boundary conditions below.

Find $\lambda \in \mathbb{R}$ and $u \in \mathrm{H}^{1}(\Omega), u \neq 0$, such that

$$
a(u, v)=\lambda b(u, v) \quad \forall v \in \mathrm{H}^{1}(\Omega) .
$$

The bilinear forms $a: \mathrm{H}^{1}(\Omega) \times \mathrm{H}^{1}(\Omega) \longrightarrow \mathbb{R}$ and $b: \mathrm{L}^{2}(\Omega) \times \mathrm{L}^{2}(\Omega) \longrightarrow \mathbb{R}$ in the equation above are defined by

$$
\begin{aligned}
a(v, w) & :=\int_{\Omega}(\nabla v \cdot \nabla w+v w) d x \quad \forall v, w \in \mathrm{H}^{1}(\Omega), \\
b(f, g) & :=\int_{\Omega} f g d x \quad \forall f, g \in \mathrm{L}^{2}(\Omega) .
\end{aligned}
$$

It is well known that problem (2.1) attains a sequence of finite multiplicity eigenvalues satisfying

$$
1=\lambda_{0}<\lambda_{1} \leq \cdots \leq \lambda_{k} \leq \cdots, \quad \lambda_{k} \stackrel{k \rightarrow \infty}{\longrightarrow}+\infty,
$$

with corresponding $\mathrm{L}^{2}(\Omega)$-orthonormal eigenfunctions $\left\{u_{k}\right\}_{k=0}^{\infty}$ providing a Hilbert basis of $\mathrm{H}^{1}(\Omega)$.

Remark 2.1. We consider the bilinear form given by (2.2) instead of the standard one for the Laplace equations (i.e., $\left.a(u, v)=\int_{\Omega} \nabla u \cdot \nabla v d x\right)$, because the eigenvalues of both problems only differ by one and the corresponding eigenfunctions coincide. Since the same is true for the discrete problem (2.4) below, we thus avoid dealing with the standard constraints for pure Neumann problems. 


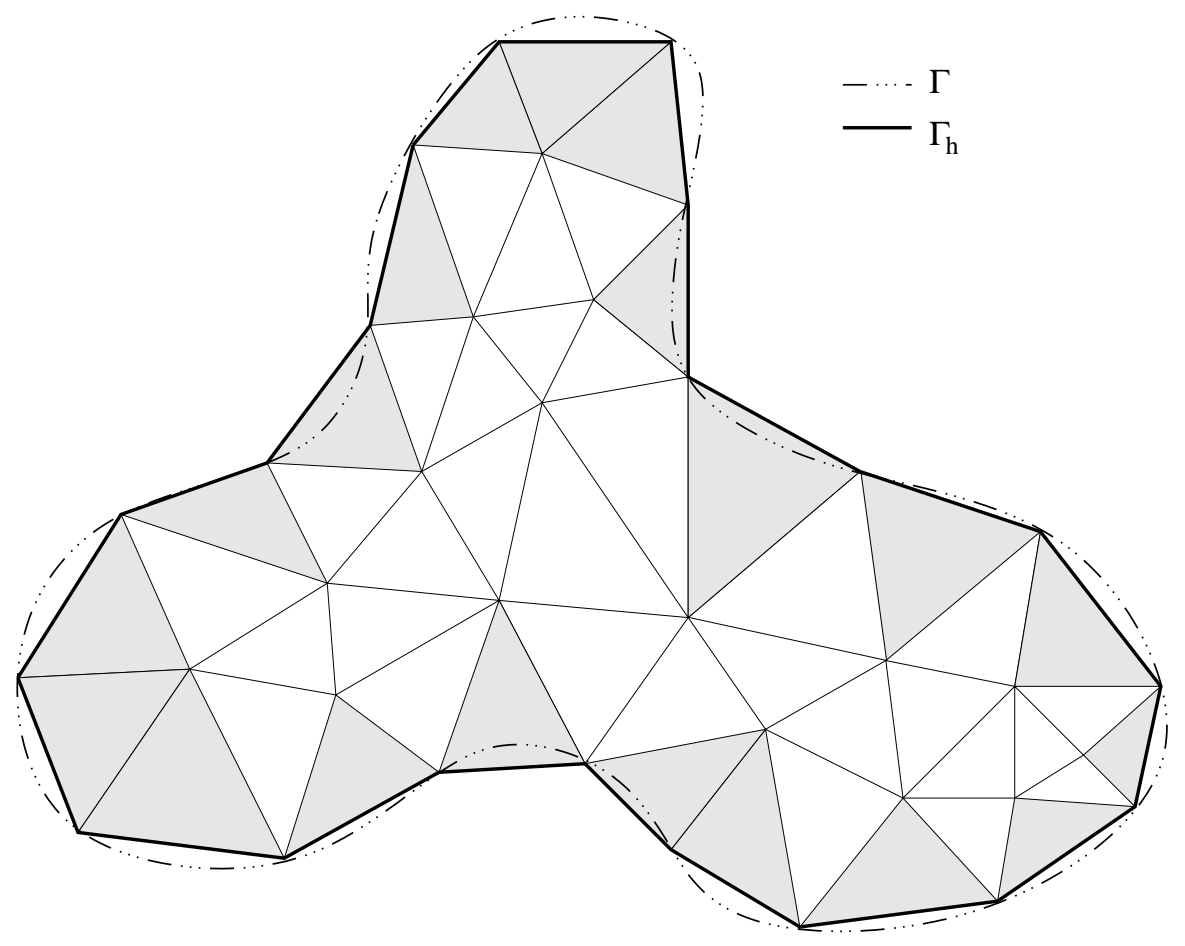

Figure 1. Triangulation of $\Omega_{h} \approx \Omega$. The shaded triangles are the boundary ones $T \in \mathcal{T}_{h}^{\partial}$.

We consider a family $\left\{\mathcal{T}_{h}\right\}$ of standard finite element triangulations (see Figure 1 and, e.g., [7) of polygonal domains $\Omega_{h}$ approximating $\Omega$, such that if $\Gamma_{h}:=\partial \Omega_{h}$ and $\mathcal{N}_{h}$ is the set of vertices of all the triangles in $\mathcal{T}_{h}$, then it holds that

- $\mathcal{N}_{h} \subset \bar{\Omega}_{h}$ and $\mathcal{N}_{h} \cap \Gamma_{h} \subset \Gamma$;

- for all $T \in \mathcal{T}_{h}$, at most two vertices of $T$ lie on $\Gamma_{h}$,

where the second assumption is only made for the sake of simplicity.

As usual, $h$ stands for the mesh-size, namely, the maximum diameter $h_{T}$ of all the triangles $T \in \mathcal{T}_{h}$. We also assume that the family $\left\{\mathcal{T}_{h}\right\}$ is regular in the sense of a minimum angle condition; i.e., there exists $\theta_{0}>0$ such that, if $\theta_{T}$ is the smallest angle of $T$, then $\theta_{T}>\theta_{0}$ for all $T \in \mathcal{T}_{h}$ and for all the triangulations $\mathcal{T}_{h}$ in the family.

In what follows, we will use the notation and definition introduced in [11] (see also [22]). For a given triangulation $\mathcal{T}_{h}$, we denote by $\mathcal{T}_{h}^{\partial}$ the subset of the so called boundary triangles; namely, those having an edge on $\Gamma_{h}$ (see Figure 1). For one such triangle $T$, let $P_{1}^{T}, P_{2}^{T}$, and $P_{3}^{T}$ be its three vertices, with $S_{T}:=\overline{P_{2}^{T} P_{3}^{T}} \subset \Gamma_{h}$, and let $\Sigma_{T}$ be the piece of $\Gamma$ approximated by $S_{T}$ (see Figure 2).

We denote by $\widetilde{T}$ the curved triangle of edges $\overline{P_{1}^{T} P_{2}^{T}}, \Sigma_{T}$, and $\overline{P_{3}^{T} P_{1}^{T}}$, and we call it the ideal triangle associated to $T$. For inner triangles $T \in \mathcal{T}_{h} \backslash \mathcal{T}_{h}^{\partial}$ we simply define $\widetilde{T} \equiv T$ as its associated ideal triangle. We denote by $\widetilde{\mathcal{T}}_{h}:=\left\{\widetilde{T}: T \in \mathcal{T}_{h}\right\}$, the partition of $\Omega$ provided by the ideal triangles and we call it the ideal triangulation 

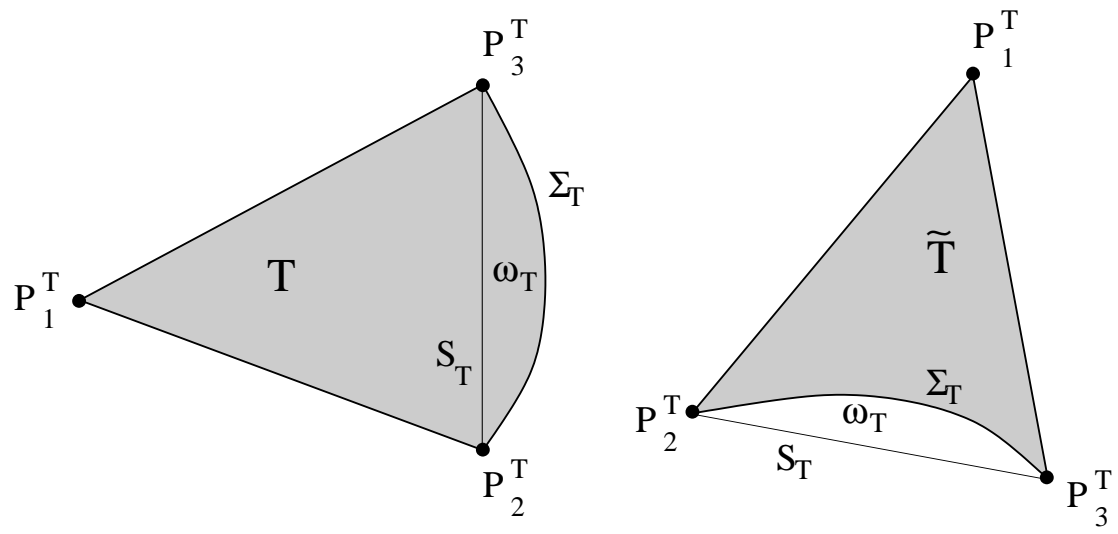

Figure 2. Ideal triangles $\widetilde{T}$.
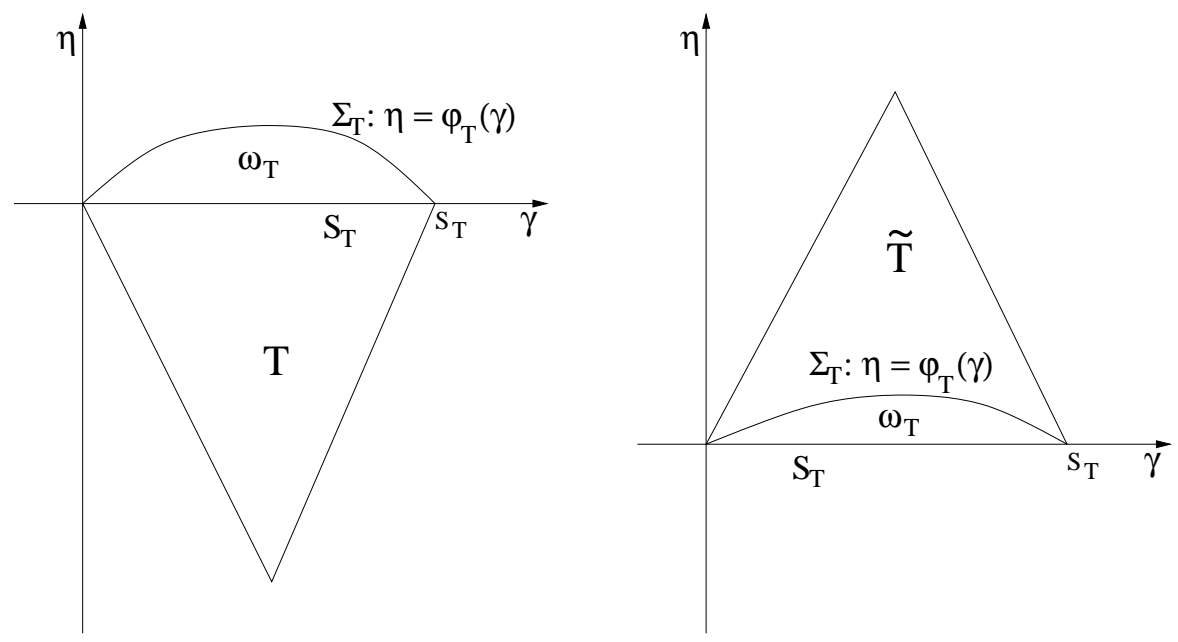

Figure 3. Local coordinates $(\gamma, \eta)$.

of $\Omega$. We also denote $\widetilde{\mathcal{T}}_{h}^{\partial}:=\left\{\widetilde{T}: T \in \mathcal{T}_{h}^{\partial}\right\}$ the family of ideal triangles associated to the boundary ones.

For the sake of simplicity we assume that the triangulations $\mathcal{T}_{h}$ are such that for each boundary triangles $T \in \mathcal{T}_{h}^{\partial}$, either $T \subset \widetilde{T}$ or $T \supset \widetilde{T}$. Let $\omega_{T}$ be the domain bounded by $\Sigma_{T}$ and $S_{T}$ (see Figure 21). Therefore $\bigcup_{T \in \mathcal{T}_{h} \text { o }} \omega_{T}=\left(\Omega \backslash \bar{\Omega}_{h}\right) \cup\left(\Omega_{h} \backslash \bar{\Omega}\right)$.

We introduce in each domain $\omega_{T}$ local coordinates $(\gamma, \eta)$, the first one along $S_{T}$ and the second normal to $S_{T}$. Let $\varphi_{T}(\gamma), 0 \leq \gamma \leq s_{T}:=\operatorname{length}\left(S_{T}\right)$, be a parametrization of $\Sigma_{T}$. We choose the orientation of $\eta$ in such a way that $\varphi_{T} \geq 0$. Then $\Sigma_{T}=\left\{(\gamma, \eta): \eta=\varphi_{T}(\gamma), \gamma \in\left[0, s_{T}\right]\right\}$ and $\omega_{T}=\left\{(\gamma, \eta): 0<\eta<\varphi_{T}, \eta \in\right.$ $\left.\left(0, s_{T}\right)\right\}$ (see Figure 3).

As a consequence of the assumed smoothness of $\Gamma, \varphi_{T} \in \mathcal{C}^{2}\left(\left[0, s_{T}\right]\right)$ and the following estimate holds. 
Lemma 2.2. There exists a constant $C>0$, independent of $T$, such that

$$
0 \leq \varphi_{T}(\gamma) \leq C s_{T}^{2} \leq C h_{T}^{2} \quad \forall \gamma \in\left[0, s_{T}\right]
$$

Proof. It is a direct consequence of standard interpolation results.

Here and hereafter, $C$ denotes a positive constant not necessarily the same at each occurrence, but always independent of the mesh-size $h$.

Consider the discretization of the spectral problem (2.1) obtained by using standard piecewise linear continuous finite elements on $\mathcal{T}_{h}$ below.

Find $\lambda_{h} \in \mathbb{R}$ and $u_{h} \in \mathcal{L}_{h}\left(\Omega_{h}\right), u_{h} \neq 0$, such that

$$
a_{h}\left(u_{h}, v_{h}\right)=\lambda_{h} b_{h}\left(u_{h}, v_{h}\right) \quad \forall v_{h} \in \mathcal{L}_{h}\left(\Omega_{h}\right) .
$$

In the equation above

$$
\mathcal{L}_{h}\left(\Omega_{h}\right):=\left\{v_{h} \in \mathrm{H}^{1}\left(\Omega_{h}\right):\left.v_{h}\right|_{T} \in \mathcal{P}_{1}(T) \forall T \in \mathcal{T}_{h}\right\},
$$

with $\mathcal{P}_{1}(T)$ being the space of linear functions defined on $T$, and the bilinear forms $a_{h}: \mathrm{H}^{1}\left(\Omega_{h}\right) \times \mathrm{H}^{1}\left(\Omega_{h}\right) \longrightarrow \mathbb{R}$ and $b_{h}: \mathrm{L}^{2}\left(\Omega_{h}\right) \times \mathrm{L}^{2}\left(\Omega_{h}\right) \longrightarrow \mathbb{R}$ are defined by

$$
\begin{aligned}
a_{h}(v, w) & :=\int_{\Omega_{h}}(\nabla v \cdot \nabla w+v w) d x \quad \forall v, w \in \mathrm{H}^{1}\left(\Omega_{h}\right), \\
b_{h}(f, g) & :=\int_{\Omega_{h}} f g d x \quad \forall f, g \in \mathrm{L}^{2}\left(\Omega_{h}\right) .
\end{aligned}
$$

Problem (2.4) reduces to a finite dimensional generalized eigenvalue problem with symmetric positive definite matrices. It attains a finite number of eigenvalues

$$
1=\lambda_{h 1}<\lambda_{h 2} \leq \cdots \leq \lambda_{h m}
$$

with corresponding $\mathrm{L}^{2}\left(\Omega_{h}\right)$-orthonormal eigenfunctions $\left\{u_{h k}\right\}_{k=0}^{m}$.

The following three basic lemmas will be used in the rest of this paper.

Lemma 2.3. There exist positive constants $c$ and $C$ (not depending on $T$ ) such that, if $v_{h} \in \mathcal{P}_{1}\left(\mathbb{R}^{2}\right)$ and $h_{T}$ is small enough, then

$$
\begin{aligned}
& c\left\|v_{h}\right\|_{0, \widetilde{T}} \leq\left\|v_{h}\right\|_{0, T} \leq C\left\|v_{h}\right\|_{0, \widetilde{T}}, \\
& c\left|v_{h}\right|_{1, \widetilde{T}} \leq\left|v_{h}\right|_{1, T} \leq C\left|v_{h}\right|_{1, \widetilde{T}} .
\end{aligned}
$$

Proof. Similar results have been proved in several papers (see, e.g., [11]). For the sake of completeness, we include an elementary proof in the case $\widetilde{T} \subset T$; the case $\widetilde{T} \supset T$ can be dealt with almost identically.

We use the notation in Figure 4. In particular $T^{\prime}$ is the triangle of vertices $P_{1}^{T}$, $Q_{2}^{T}$, and $Q_{3}^{T}$, with $Q_{k}^{T}$ being the midpoint of the edge $\overline{P_{1}^{T} P_{k}^{T}}$, for $k=2,3$.

Because of Lemma 2.2 for $h_{T}$ small enough, the triangle $T^{\prime}$ is contained in $\widetilde{T}$ as shown in Figure 4. Then straightforward computations show that $\left\|v_{h}\right\|_{0, T} \leq$ $C\left\|v_{h}\right\|_{0, T^{\prime}}$ and $\left|v_{h}\right|_{1, T}=2\left|v_{h}\right|_{1, \tilde{T}}$. Hence, the lemma is a consequence of the fact that $T^{\prime} \subset \widetilde{T} \subset T$.

Lemma 2.4. There exists a positive constant $C$ such that if $h$ is small enough, then

$$
\begin{aligned}
\|v\|_{0, \Omega \backslash \bar{\Omega}_{h}} & \leq C h^{s}\|v\|_{s, \Omega} \quad \forall v \in \mathrm{H}^{s}(\Omega) \quad(0 \leq s \leq 1) \\
\|v\|_{0, \Omega_{h} \backslash \bar{\Omega}} & \leq C h^{s}\|v\|_{s, \Omega_{h}} \quad \forall v \in \mathrm{H}^{s}\left(\Omega_{h}\right) \quad(0 \leq s \leq 1) .
\end{aligned}
$$




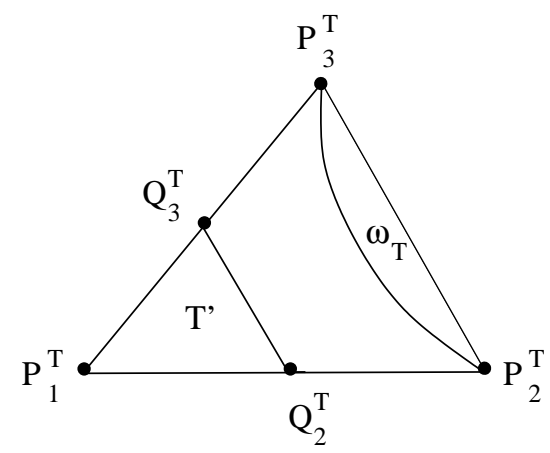

Figure 4. Auxiliary triangle $T^{\prime}($ case: $\widetilde{T} \subset T)$.

Proof. The two inequalities have been essentially proved in the proof of Lemma $5.2-5.3$ in [16] for $s=1$. Since the inequalities are clearly true for $s=0$, they follow for $0<s<1$ from standard results on interpolation in Sobolev spaces (see, for instance, Theorem 1.4 in [12]).

Lemma 2.5. There exists a positive constant $C$ such that if $h_{T}$ is small enough, then, for all $T \in \mathcal{T}_{h}$,

$$
\begin{array}{llll}
\|v\|_{0, \widetilde{T} \backslash T} \leq C\left(\|v\|_{0, T}+h_{T}^{2 s}\|v\|_{s, \widetilde{T}}\right) & \forall v \in \mathrm{H}^{s}(\widetilde{T}) & (0 \leq s \leq 1), & \text { if } T \subset \widetilde{T}, \\
\|v\|_{0, T \backslash \widetilde{T}} \leq C\left(\|v\|_{0, \widetilde{T}}+h_{T}^{2 s}\|v\|_{s, T}\right) & \forall v \in \mathrm{H}^{s}(T) & (0 \leq s \leq 1), & \text { if } \widetilde{T} \subset T .
\end{array}
$$

Proof. We only include the proof of the first inequality. The second one can be obtained by applying similar arguments. We use the notation shown in Figure 5 for $T \subset \widetilde{T}$.

Consider polar coordinates centered at the vertex $P_{1}^{T}$. We assume that $S_{T}$ and $\Sigma_{T}$ are parametrized by $r=r_{1}(\theta)$ and $r=r_{2}(\theta)$, respectively, with $0 \leq \theta \leq \theta_{1}$. Then $\omega_{T}:=\widetilde{T} \backslash T=\left\{(r, \theta): r_{1}(\theta)<r<r_{2}(\theta), 0<\theta<\theta_{1}\right\}$. Let $d$ denote the distance of each point on $\Sigma_{T}$ to $S_{T}$, as shown in Figure 5 .

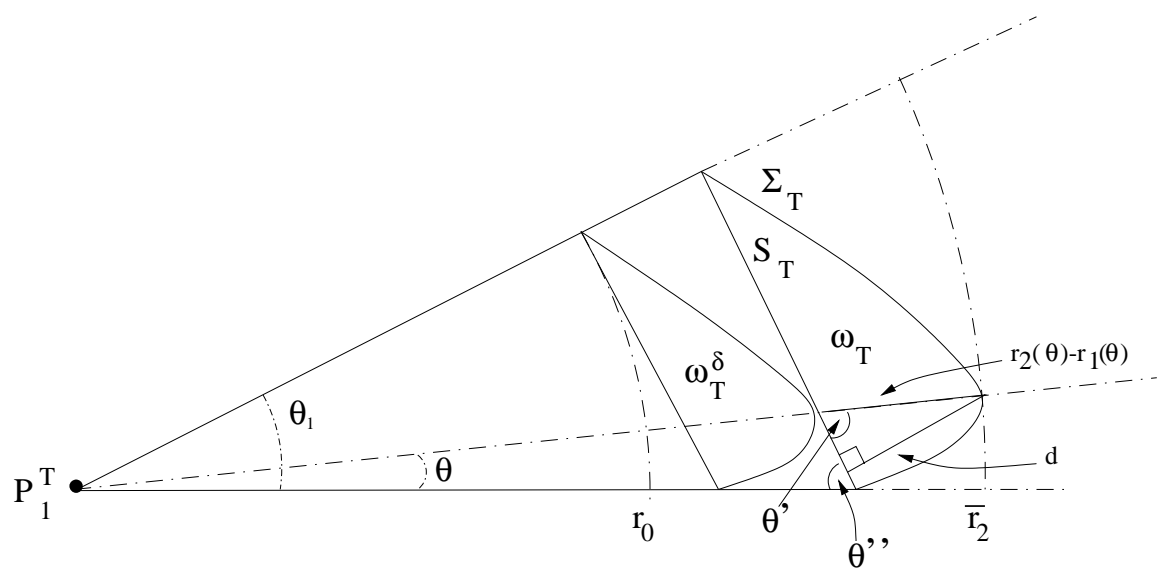

Figure 5. Polar coordinates on $\widetilde{T} \supset T$. 
Assume that $\theta^{\prime} \leq \frac{\pi}{2}$ as in Figure 5 (a similar argument is valid for $\theta^{\prime} \geq \frac{\pi}{2}$ ). Since $\theta^{\prime}=\theta^{\prime \prime}+\theta$, then $\sin \theta^{\prime} \geq \sin \theta^{\prime \prime} \geq \sin \theta_{T}$, with $\theta_{T}$ being the smallest angle of $T$. Hence,

$$
r_{2}(\theta)-r_{1}(\theta)=\frac{d}{\sin \theta^{\prime}} \leq \frac{d}{\sin \theta_{T}} .
$$

Then, because of Lemma 2.2 and the minimum angle condition,

$$
\delta:=\max _{0 \leq \theta \leq \theta_{1}}\left[r_{2}(\theta)-r_{1}(\theta)\right] \leq C h_{T}^{2},
$$

and, consequently, for $h_{T}$ small enough,

$$
\begin{aligned}
& r_{0}:=\min _{0 \leq \theta \leq \theta_{1}} r_{1}(\theta)-\delta \geq c h_{T}>0, \\
& \bar{r}_{2}:=\max _{0 \leq \theta \leq \theta_{1}} r_{2}(\theta) \leq C h_{T} .
\end{aligned}
$$

Hence, for $h_{T}$ small enough, $\omega_{T}^{\delta}:=\left\{(r-\delta, \theta):(r, \theta) \in \omega_{T}\right\} \subset T$ (see Figure 51).

Let $v^{\delta}(r, \theta):=v(r-\delta, \theta)$, for $(r, \theta) \in \omega_{T}$. We will prove that

$$
\left\|v-v^{\delta}\right\|_{0, \omega_{T}} \leq C h_{T}^{2 s}\|v\|_{s, \widetilde{T}}
$$

Then the lemma follows from this inequality and the fact that, for $h_{T}$ small enough, $\left\|v^{\delta}\right\|_{0, \omega_{T}} \leq C\|v\|_{0, \omega_{T}^{\delta}} \leq C\|v\|_{0, T}$.

Inequality (2.7) is clear for $s=0$. We prove it for $s=1$; then, for $0<s<1$, it follows by interpolation between Sobolev spaces.

Let $v \in \mathcal{C}^{\infty}(\widetilde{T})$ (we prove (2.7) for such smooth $v$; for any $v \in \mathrm{H}^{1}(\widetilde{T})$ it follows by the standard density argument). For any $(r, \theta) \in \omega_{T}$ we may write

$$
v(r, \theta)-v^{\delta}(r, \theta)=\int_{r-\delta}^{r} \frac{\partial v}{\partial r}(t, \theta) d t .
$$

Hence, applying the Cauchy-Schwarz inequality and integrating on $\omega_{T}$, we obtain

$$
\begin{aligned}
\left\|v-v^{\delta}\right\|_{0, \omega_{T}}^{2} & \leq \int_{0}^{\theta_{1}} \int_{r_{1}(\theta)}^{r_{2}(\theta)} \delta \int_{r-\delta}^{r}\left|\frac{\partial v}{\partial r}(t, \theta)\right|^{2} d t r d r d \theta \\
& \leq \delta \int_{0}^{\theta_{1}}\left[\int_{r_{0}}^{r_{2}(\theta)}\left|\frac{\partial v}{\partial r}(t, \theta)\right|^{2} \frac{t}{r_{0}} d t\right]\left[\int_{r_{1}(\theta)}^{r_{2}(\theta)} r d r\right] d \theta \\
& \leq \delta \int_{0}^{\theta_{1}}\left[\int_{0}^{r_{2}(\theta)}|\nabla v(t, \theta)|^{2} t d t\right] \frac{2 \bar{r}_{2}}{r_{0}}\left[r_{2}(\theta)-r_{1}(\theta)\right] d \theta \\
& \leq C \delta^{2}\|\nabla v\|_{0, \widetilde{T}}^{2} .
\end{aligned}
$$

Thus, for $s=1$, (2.7) is a consequence of the fact that $\delta \leq C h_{T}^{2}$.

Consider the operator $\mathbf{T}$ defined by

$$
\begin{aligned}
\mathbf{T}: \mathrm{L}^{2}(\Omega) & \longrightarrow \mathrm{L}^{2}(\Omega) \\
f & \longmapsto u \in \mathrm{H}^{1}(\Omega): a(u, v)=b(f, v) \quad \forall v \in \mathrm{H}^{1}(\Omega),
\end{aligned}
$$

where $a(\cdot, \cdot)$ and $b(\cdot, \cdot)$ are the bilinear forms defined in (2.2) and (2.3), respectively. By virtue of the Lax-Milgram lemma, it is clear that $\mathbf{T}$ is a well-defined bounded operator and

$$
\|u\|_{1, \Omega} \leq C\|f\|_{0, \Omega}
$$


Furthermore, because of the compact inclusion $\mathrm{H}^{1}(\Omega) \hookrightarrow \mathrm{L}^{2}(\Omega)$, $\mathbf{T}$ is compact. Clearly, the eigenvalues of $\mathbf{T}$ are given by $\mu_{k}=1 / \lambda_{k}$, with $\lambda_{k}$ being the eigenvalues of problem (2.1), and the corresponding eigenfunctions $u_{k}$ coincide.

As a consequence of the classical a priori estimates (see [13]), for any $f \in \mathrm{L}^{2}(\Omega)$, $u=\mathbf{T} f$ is known to satisfy some further regularity. In fact, $u \in \mathrm{H}^{1+r}(\Omega)$ for some $r>0$ depending on the geometry of $\Omega$, and there holds

$$
\|u\|_{1+r, \Omega} \leq C\|f\|_{0, \Omega} .
$$

For a pure Neumann problem, if $\Omega$ has no reentrant corners, then $r=1$; otherwise, $r<\frac{2 \pi}{\theta}$, with $\theta$ being the largest interior angle of $\Omega$. From now on let $r \in(0,1]$ be fixed such that (2.9) holds. Notice that, as a consequence, the eigenfunctions $u_{k}$ of $\mathbf{T}$ belong to $\mathrm{H}^{1+r}(\Omega)$ and satisfy

$$
\left\|u_{k}\right\|_{1+r, \Omega} \leq C\left\|u_{k}\right\|_{0, \Omega} .
$$

Let $\overline{\mathbf{T}}_{h}$ be defined by

$$
\begin{aligned}
\overline{\mathbf{T}}_{h}: \mathrm{L}^{2}\left(\Omega_{h}\right) & \longrightarrow \mathrm{L}^{2}\left(\Omega_{h}\right) \\
g & \longmapsto v_{h} \in \mathcal{L}_{h}\left(\Omega_{h}\right): a_{h}\left(v_{h}, w_{h}\right)=b_{h}\left(g, w_{h}\right) \quad \forall w_{h} \in \mathcal{L}_{h}\left(\Omega_{h}\right),
\end{aligned}
$$

with $a_{h}(\cdot, \cdot)$ and $b_{h}(\cdot, \cdot)$ as defined in (2.5) and (2.6), respectively. Clearly

$$
\left\|v_{h}\right\|_{1, \Omega_{h}} \leq C\|g\|_{0, \Omega_{h}} \text {. }
$$

The positive nonzero eigenvalues of $\overline{\mathbf{T}}_{h}$ are given by $\mu_{h k}=1 / \lambda_{h k}$, with $\lambda_{h k}$ being those of the discrete problem (2.4) and coinciding associated eigenfunctions $u_{h k}$.

The spectral approximation theory stated in [1] cannot be directly applied to the operators $\overline{\mathbf{T}}_{h}$, since their domains $\mathrm{L}^{2}\left(\Omega_{h}\right)$ do not coincide with that of $\mathbf{T}$. So, we are going to introduce other discrete operators $\mathbf{T}_{h}$ defined on $\mathrm{L}^{2}(\Omega)$ and with spectrum also related to that of problem (2.4).

From now on, we assume that $h$ is sufficiently small in order for Lemmas 2.3 . 2.4, and 2.5 to hold. Let

$$
\mathcal{L}_{h}(\Omega):=\left\{v_{h} \in \mathrm{H}^{1}(\Omega):\left.v_{h}\right|_{\widetilde{T}} \in \mathcal{P}_{1}(\widetilde{T}) \forall \widetilde{T} \in \widetilde{\mathcal{T}}_{h}\right\} .
$$

We consider two restriction-extension operators, one from $\mathcal{L}_{h}\left(\Omega_{h}\right)$ to $\mathcal{L}_{h}(\Omega)$ and the other from $\mathcal{L}_{h}(\Omega)$ to $\mathcal{L}_{h}\left(\Omega_{h}\right)$. Let

$$
\begin{aligned}
\check{\mathbf{E}}: \mathcal{L}_{h}\left(\Omega_{h}\right) & \longrightarrow \mathcal{L}_{h}(\Omega) \\
v_{h} & \longmapsto \check{v}_{h}
\end{aligned}
$$

with

$$
\left.\check{v}_{h}\right|_{\widetilde{T}}:= \begin{cases}\left.v_{h}\right|_{\widetilde{T}} \quad \forall \widetilde{T} \in \widetilde{\mathcal{T}}_{h}: \widetilde{T} \subset \Omega_{h}, \\ \left(\left.v_{h}\right|_{T}\right)^{\sim} \quad \forall \widetilde{T} \in \widetilde{\mathcal{T}}_{h}: \widetilde{T} \not \subset \Omega_{h},\end{cases}
$$

where $\left(\left.v_{h}\right|_{T}\right)^{\sim} \in \mathcal{P}_{1}(\widetilde{T})$ denotes the natural extension of the linear function $\left.v_{h}\right|_{T} \in$ $\mathcal{P}_{1}(T)$ to the larger set $\widetilde{T}$ (notice that, if $\widetilde{T} \not \subset \Omega_{h}$, then $T \subset \widetilde{T}$ ).

Let $\hat{\mathbf{E}}:=\check{\mathbf{E}}^{-1}$; namely,

$$
\begin{aligned}
\hat{\mathbf{E}}: \mathcal{L}_{h}(\Omega) & \longrightarrow \mathcal{L}_{h}\left(\Omega_{h}\right) \\
w_{h} & \longmapsto \hat{w}_{h}
\end{aligned}
$$

with

$$
\left.\hat{w}_{h}\right|_{T}:= \begin{cases}\left.w_{h}\right|_{T} \quad \forall T \in \mathcal{T}_{h}: T \subset \Omega, \\ \left(\left.w_{h}\right|_{\widetilde{T}}\right)^{\wedge} \quad \forall T \in \mathcal{T}_{h}: T \not \subset \Omega,\end{cases}
$$


where $\left(\left.w_{h}\right|_{\widetilde{T}}\right)^{\wedge} \in \mathcal{P}_{1}(T)$ denotes the natural extension of the linear function $\left.w_{h}\right|_{\widetilde{T}} \in$ $\mathcal{P}_{1}(\widetilde{T})$ to the larger set $T$ (notice that now if $T \not \subset \Omega$, then $\widetilde{T} \subset T$ ).

As a consequence of Lemma 2.3 the operators $\check{\mathbf{E}}$ and $\hat{\mathbf{E}}$ are uniformly bounded in $\mathrm{L}^{2}$ and $\mathrm{H}^{1}$ norms. More precisely,

$$
\begin{array}{rlrl}
\left\|\check{\mathbf{E}} v_{h}\right\|_{k, \Omega} \leq C\left\|v_{h}\right\|_{k, \Omega_{h}} & \forall v_{h} \in \mathcal{L}_{h}\left(\Omega_{h}\right), & k & =0,1, \\
\left\|\hat{\mathbf{E}} w_{h}\right\|_{k, \Omega_{h}} \leq C\left\|w_{h}\right\|_{k, \Omega} & \forall w_{h} \in \mathcal{L}_{h}(\Omega), & k=0,1 .
\end{array}
$$

We will also use the $\mathrm{L}^{2}(\Omega)$-projection onto $\mathcal{L}_{h}(\Omega)$. Let

$$
\begin{aligned}
\mathbf{P}: \mathrm{L}^{2}(\Omega) & \longrightarrow \mathcal{L}_{h}(\Omega) \\
f & \longmapsto f_{h}
\end{aligned}
$$

with

$$
f_{h} \in \mathcal{L}_{h}(\Omega): \quad \int_{\Omega}\left(f_{h}-f\right) w_{h} d x=0 \quad \forall w_{h} \in \mathcal{L}_{h}(\Omega) .
$$

Clearly

$$
\left\|f_{h}\right\|_{0, \Omega} \leq\|f\|_{0, \Omega} \quad \forall f \in \mathrm{L}^{2}(\Omega) .
$$

Furthermore, the standard error estimate for this projection yields

$$
\left\|f_{h}-f\right\|_{0, \Omega} \leq C h\|f\|_{1, \Omega} \quad \forall f \in \mathrm{H}^{1}(\Omega) .
$$

Now we are able to define the discrete operator that we will use in the rest of this paper:

$$
\begin{aligned}
\mathbf{T}_{h}: \mathrm{L}^{2}(\Omega) & \longrightarrow \mathrm{L}^{2}(\Omega) \\
f & \longmapsto \check{u}_{h}=\check{\mathbf{E}}_{h} \hat{\mathbf{E}} \mathbf{P} f
\end{aligned}
$$

As a consequence of the estimates (2.15), (2.13), (2.11), and (2.12), the operators $\mathbf{T}_{h}$ are uniformly bounded. Moreover, they satisfy

$$
\left\|\mathbf{T}_{h} f\right\|_{1, \Omega} \leq C\|f\|_{0, \Omega}
$$

The following lemma shows that the eigenvalues of $\mathbf{T}_{h}$ and $\overline{\mathbf{T}}_{h}$ coincide and the eigenfunctions of one operator are the restriction-extension of those of the other.

Lemma 2.6. The nonzero eigenvalues of $\overline{\mathbf{T}}_{h}$ and $\mathbf{T}_{h}$ coincide and the respective associated eigenfunctions $u_{h}$ and $\check{u}_{h}$ are related by $\check{u}_{h}=\check{\mathbf{E}} u_{h}$ and $u_{h}=\hat{\mathbf{E}} \check{u}_{h}$.

Proof. Let $u_{h} \in \mathcal{L}_{h}\left(\Omega_{h}\right), u_{h} \neq 0$, such that $\overline{\mathbf{T}}_{h} u_{h}=\lambda_{h} u_{h}$. Then

$$
\mathbf{T}_{h} \check{\mathbf{E}} u_{h}=\check{\mathbf{E}} \overline{\mathbf{T}}_{h} \hat{\mathbf{E}} \mathbf{P} \check{\mathbf{E}} u_{h}=\check{\mathbf{E}} \overline{\mathbf{T}}_{h} \hat{\mathbf{E}} \check{\mathbf{E}} u_{h}=\check{\mathbf{E}} \overline{\mathbf{T}}_{h} u_{h}=\lambda_{h} \check{\mathbf{E}} u_{h} \text {. }
$$

Conversely, let $\check{u}_{h} \in \mathcal{L}_{h}(\Omega), \check{u}_{h} \neq 0$, such that $\mathbf{T}_{h} \check{u}_{h}=\lambda_{h} \check{u}_{h}$. Then

$$
\overline{\mathbf{T}}_{h} \hat{\mathbf{E}} \check{u}_{h}=\hat{\mathbf{E}} \check{\mathbf{E}} \overline{\mathbf{T}}_{h} \hat{\mathbf{E}} \mathbf{P} \check{u}_{h}=\hat{\mathbf{E}} \mathbf{T}_{h} \check{u}_{h}=\lambda_{h} \hat{\mathbf{E}} \check{u}_{h} .
$$

The aim of this paper is to study how the eigenvalues and eigenfunctions of $\mathbf{T}_{h}$, approximate those of $\mathbf{T}$, and hence to analyze how the eigenvalue problem (2.4) allows approximating the solutions of the spectral problem (2.1). 


\section{Spectral approximation}

In order to use the spectral approximation theory stated in [1], we are going to prove that the operators $\mathbf{T}_{h}$ converge to $\mathbf{T}$ in norm as $h$ goes to zero. From now on and throughout the rest of the section, we use the following notation: given $f \in \mathrm{L}^{2}(\Omega)$, we denote $u:=\mathbf{T} f, f_{h}:=\mathbf{P} f, \hat{f}_{h}:=\hat{\mathbf{E}} f_{h}, u_{h}:=\overline{\mathbf{T}}_{h} \hat{f}_{h}$, and $\check{u}_{h}:=\check{\mathbf{E}} u_{h}=\mathbf{T}_{h} f$.

In the proofs, we will also use extensions to $\Omega \cup \Omega_{h}$ of functions originally defined in $\Omega$. For $v \in \mathrm{H}^{s}(\Omega)(s>0)$, let $v^{e}$ denote an extension of $v$ to $\mathbb{R}^{2}$ satisfying (see, for instance, Theorem 1.4.3.1 in [13])

$$
\left\|v^{e}\right\|_{s, \mathbb{R}^{2}} \leq C\|v\|_{s, \Omega} .
$$

In particular since, according to the estimate (2.9), $u \in \mathrm{H}^{1+r}(\Omega)$, let $u^{e}$ be an extension of $u$ satisfying

$$
\left\|u^{e}\right\|_{1+r, \mathbb{R}^{2}} \leq C\|u\|_{1+r, \Omega}
$$

The following lemma splits $\left\|\left(\mathbf{T}-\mathbf{T}_{h}\right) f\right\|_{1, \Omega}=\left\|u-\check{u}_{h}\right\|_{1, \Omega}$ into three terms which can be dealt with separately.

Lemma 3.1. There exists a positive constant $C$, not depending on $f$, such that

$$
\begin{aligned}
\left\|u-\check{u}_{h}\right\|_{1, \Omega} \leq C & {\left[\inf _{v_{h} \in \mathcal{L}_{h}\left(\Omega_{h}\right)}\left\|u^{e}-v_{h}\right\|_{1, \Omega_{h}}\right.} \\
& \left.+\sup _{w_{h} \in \mathcal{L}_{h}\left(\Omega_{h}\right)} \frac{\left|b_{h}\left(\hat{f}_{h}, w_{h}\right)-a_{h}\left(u^{e}, w_{h}\right)\right|}{\left\|w_{h}\right\|_{1, \Omega_{h}}}\right]+\left\|u-\check{u}_{h}\right\|_{1, \Omega \backslash \bar{\Omega}_{h}} .
\end{aligned}
$$

Proof. It is enough to notice that

$$
\begin{aligned}
\left\|u-\check{u}_{h}\right\|_{1, \Omega}^{2} & =\left\|u-u_{h}\right\|_{1, \Omega \cap \Omega_{h}}^{2}+\left\|u-\check{u}_{h}\right\|_{1, \Omega \backslash \bar{\Omega}_{h}}^{2} \\
& \leq\left\|u-u_{h}\right\|_{1, \Omega_{h}}^{2}+\left\|u-\check{u}_{h}\right\|_{1, \Omega \backslash \bar{\Omega}_{h}}^{2},
\end{aligned}
$$

and to use the standard techniques to deal with nonconforming methods to estimate the first term (see Strang's lemma, for instance, in [7]):

$$
\left\|u^{e}-u_{h}\right\|_{1, \Omega_{h}} \leq 2 \inf _{v_{h} \in \mathcal{L}_{h}\left(\Omega_{h}\right)}\left\|u^{e}-v_{h}\right\|_{1, \Omega_{h}}+\sup _{w_{h} \in \mathcal{L}_{h}\left(\Omega_{h}\right)} \frac{\left|b_{h}\left(\hat{f}_{h}, w_{h}\right)-a_{h}\left(u^{e}, w_{h}\right)\right|}{\left\|w_{h}\right\|_{1, \Omega_{h}}} .
$$

In what follows we give estimates of the three terms in the right-hand side of (3.3). For the first one we have

Lemma 3.2. There exists a positive constant $C$, not depending on $f$, and $v_{h} \in$ $\mathcal{L}_{h}\left(\Omega_{h}\right)$ such that

$$
\left\|u^{e}-v_{h}\right\|_{1, \Omega_{h}} \leq C h^{r}\|f\|_{0, \Omega} .
$$

Proof. Let $v_{h} \in \mathcal{L}_{h}\left(\Omega_{h}\right)$ be the Lagrange interpolant of $u^{e} \in \mathrm{H}^{1+r}\left(\Omega_{h}\right)$. Proceeding as in Example 3 of [9] we have

$$
\left\|u^{e}-v_{h}\right\|_{1, \Omega_{h}} \leq C h^{r}\left\|u^{e}\right\|_{1+r, \Omega_{h}}
$$

(see also Theorem 2.27 in [10] for an alternative proof). So, the lemma follows from this inequality and estimates (3.2) and (2.9). 
Now we estimate the second term in the right-hand side of (3.3).

Lemma 3.3. There exists a positive constant $C$, not depending on $f$, such that

$$
\sup _{w_{h} \in \mathcal{L}_{h}\left(\Omega_{h}\right)} \frac{\left|b_{h}\left(\hat{f}_{h}, w_{h}\right)-a_{h}\left(u^{e}, w_{h}\right)\right|}{\left\|w_{h}\right\|_{1, \Omega_{h}}} \leq C h^{r}\|f\|_{0, \Omega} .
$$

Proof. Let $w_{h} \in \mathcal{L}_{h}\left(\Omega_{h}\right)$ and $\check{w}_{h}=\check{\mathbf{E}} w_{h}$. Since $\check{w}_{h} \in \mathrm{H}^{1}(\Omega)$, then $a\left(u, \check{w}_{h}\right)=$ $b\left(f, \check{w}_{h}\right)$, and hence

$$
b_{h}\left(\hat{f}_{h}, w_{h}\right)-a_{h}\left(u^{e}, w_{h}\right)=\left[b_{h}\left(\hat{f}_{h}, w_{h}\right)-b\left(f, \check{w}_{h}\right)\right]+\left[a\left(u, \check{w}_{h}\right)-a_{h}\left(u^{e}, w_{h}\right)\right] .
$$

Because of (2.14) and the fact that $\check{w}_{h} \in \mathcal{L}_{h}(\Omega)$, we have

$$
\begin{aligned}
b_{h}\left(\hat{f}_{h}, w_{h}\right)-b\left(f, \check{w}_{h}\right) & =\int_{\Omega_{h}} \hat{f}_{h} w_{h} d x-\int_{\Omega} f \check{w}_{h} d x \\
& =\int_{\Omega_{h} \backslash \bar{\Omega}} \hat{f}_{h} w_{h} d x-\int_{\Omega \backslash \bar{\Omega}_{h}} f_{h} \check{w}_{h} d x .
\end{aligned}
$$

Analogously,

$$
\begin{aligned}
a\left(u, \check{w}_{h}\right)-a_{h}\left(u^{e}, w_{h}\right)= & \int_{\Omega \backslash \bar{\Omega}_{h}}\left(\nabla u \cdot \nabla \check{w}_{h}+u \check{w}_{h}\right) d x \\
& -\int_{\Omega_{h} \backslash \bar{\Omega}}\left(\nabla u^{e} \cdot \nabla w_{h}+u^{e} w_{h}\right) d x .
\end{aligned}
$$

Now by using the Cauchy-Schwarz inequality and Lemma 2.4 we obtain

$$
\begin{aligned}
&\left|\int_{\Omega_{h} \backslash \bar{\Omega}} \hat{f}_{h} w_{h} d x\right| \leq\left\|\hat{f}_{h}\right\|_{0, \Omega_{h} \backslash \bar{\Omega}} C h\left\|w_{h}\right\|_{1, \Omega_{h}}, \\
&\left|\int_{\Omega^{\prime} \backslash \bar{\Omega}_{h}} f_{h} \check{w}_{h} d x\right| \leq\left\|f_{h}\right\|_{0, \Omega \backslash \bar{\Omega}_{h}} C h\left\|\check{w}_{h}\right\|_{1, \Omega}, \\
&\left|\int_{\Omega \backslash \bar{\Omega}_{h}}\left(\nabla u \cdot \nabla \check{w}_{h}+u \check{w}_{h}\right) d x\right| \leq C h^{r}\|u\|_{1+r, \Omega}\left\|\check{w}_{h}\right\|_{1, \Omega \backslash \bar{\Omega}_{h}}, \\
&\left|\int_{\Omega_{h} \backslash \bar{\Omega}}\left(\nabla u^{e} \cdot \nabla w_{h}+u^{e} w_{h}\right) d x\right| \leq C h^{r}\left\|u^{e}\right\|_{1+r, \Omega_{h}}\left\|w_{h}\right\|_{1, \Omega_{h} \backslash \bar{\Omega}} .
\end{aligned}
$$

Finally, we conclude the lemma bounding the four right-hand sides above by $C h^{r}\|f\|_{0, \Omega}\left\|w_{h}\right\|_{1, \Omega_{h}}$. To do this, we use (2.13) and (2.15) for the first one, (2.12) for the second one, (2.9) and (2.12) for the third one, and (3.2) and (2.9) for the last one.

Remark 3.4. Combining estimate (3.4) in the proof of Lemma 3.1, with Lemmas 3.2 and 3.3 we also have

$$
\left\|u^{e}-u_{h}\right\|_{1, \Omega_{h}} \leq C h^{r}\|f\|_{0, \Omega} .
$$

Now we are able to estimate the third term in (3.3).

Lemma 3.5. There exists a positive constant $C$, not depending on $f$, such that

$$
\left\|u-\check{u}_{h}\right\|_{1, \Omega \backslash \bar{\Omega}_{h}} \leq C h^{r}\|f\|_{0, \Omega} .
$$


Proof. For the $\mathrm{L}^{2}$ norm, we use Lemma 2.4 and estimates (2.8) and (2.17) to obtain

$$
\left\|u-\check{u}_{h}\right\|_{0, \Omega \backslash \bar{\Omega}_{h}} \leq C h\|u\|_{1, \Omega}+C h\left\|\check{u}_{h}\right\|_{1, \Omega} \leq C h\|f\|_{0, \Omega} .
$$

For the $\mathrm{H}^{1}$ seminorm we use Lemma 2.5

$$
\begin{aligned}
\left|u-\check{u}_{h}\right|_{1, \Omega \backslash \bar{\Omega}_{h}}^{2} & =\sum_{T \in \mathcal{T}_{h}^{\partial}: T \subset \widetilde{T}}\left\|\nabla\left(u-\check{u}_{h}\right)\right\|_{0, \widetilde{T} \backslash T}^{2} \\
& \leq \sum_{T \in \mathcal{T}_{h}^{\partial}: T \subset \widetilde{T}} C\left[\left\|\nabla\left(u-\check{u}_{h}\right)\right\|_{0, T}+h_{T}^{2 r}\left\|\nabla\left(u-\check{u}_{h}\right)\right\|_{r, \widetilde{T}}\right]^{2} \\
& \leq C\left[\left\|u^{e}-u_{h}\right\|_{1, \Omega_{h}}^{2}+h^{4 r}\left(\|u\|_{1+r, \Omega}^{2}+\left\|\check{u}_{h}\right\|_{1, \Omega}^{2}\right)\right],
\end{aligned}
$$

where we have used that $\left\|\nabla \check{u}_{h}\right\|_{r, \widetilde{T}}=\left\|\nabla \check{u}_{h}\right\|_{0, \widetilde{T}}$, since $\left.\check{u}_{h}\right|_{\widetilde{T}} \in \mathcal{P}_{1}(\widetilde{T})$. Therefore, the lemma follows from Remark 3.4 and estimates (2.9) and (2.17).

Now we may conclude the convergence in norm of $\mathbf{T}_{h}$ to $\mathbf{T}$.

Lemma 3.6. There exists a positive constant $C$ such that, for all $f \in \mathrm{L}^{2}(\Omega)$,

$$
\left\|\left(\mathbf{T}-\mathbf{T}_{h}\right) f\right\|_{1, \Omega} \leq C h^{r}\|f\|_{0, \Omega}
$$

Proof. It is an immediate consequence of the four previous lemmas.

Therefore, we are able to apply the spectral approximation theory for compact operators (see [1) to obtain optimal order error estimates for the eigenfunctions. For simplicity we state the result for a simple eigenvalue; see [1] for the general statement.

Theorem 3.7. Let $\mu_{k}$ be the $k$-th (simple) eigenvalue of $\mathbf{T}$ and $\mu_{h k}$ the $k$-th eigenvalue of $\mathbf{T}_{h}$. Then, the corresponding eigenfunctions $u_{k}$ and $\check{u}_{h k}$ can be chosen such that $\left\|u_{k}\right\|_{0, \Omega}=\left\|\check{u}_{h k}\right\|_{0, \Omega}=1$ and

$$
\left\|u_{k}-\check{u}_{h k}\right\|_{1, \Omega} \leq C h^{r}
$$

with $C$ a strictly positive constant.

Proof. It is a direct consequence of the convergence in norm of $\mathbf{T}_{h}$ to $\mathbf{T}$ and the error estimate in the previous lemma, and Lemma 7.1 in [1].

In the remainder of the paper we will prove optimal order error estimates for the $\mathrm{L}^{2}$ norm of the eigenfunctions and for the eigenvalues. To this end, we will use a double order error estimate for $\left\|u-\check{u}_{h}\right\|_{0, \Omega}$. To the best of the authors' knowledge, estimates of this type have not been proved for Neumann boundary conditions on curved domains. The proofs given below for the estimate of $\left\|u-\breve{u}_{h}\right\|_{0, \Omega}$ are valid only for smooth right-hand sides $f$ (namely, $f \in \mathrm{H}^{1}(\Omega)$ ). However, the results obtained suffice to yield optimal error estimates for the spectral problem without assuming any regularity.

The following two lemmas are valid for $f \in \mathrm{H}^{1}(\Omega)$. For such functions, we denote by $f^{e}$ their bounded extensions to $\mathbb{R}^{2}$ satisfying (3.1) for $s=1$; namely

$$
\left\|f^{e}\right\|_{1, \mathbb{R}^{2}} \leq C\|f\|_{1, \Omega} \text {. }
$$

First we prove the following technical result.

Lemma 3.8. There exists a positive constant $C$ such that, for all $f \in \mathrm{H}^{1}(\Omega)$,

$$
\left\|\hat{f}_{h}-f^{e}\right\|_{0, \Omega_{h} \backslash \bar{\Omega}} \leq C h\|f\|_{1, \Omega} .
$$


Proof. According to Lemma 2.5 we have $\forall T \in \mathcal{T}_{h}^{\partial}$ such that $T \supset \widetilde{T}$,

$$
\left\|\hat{f}_{h}-f^{e}\right\|_{0, T \backslash \widetilde{T}} \leq C\left(\left\|f_{h}-f\right\|_{0, \widetilde{T}}+h_{T}^{2}\left\|\hat{f}_{h}-f^{e}\right\|_{1, T}\right) .
$$

From a standard local inverse inequality (see for instance [7]), we obtain

$$
\left|\hat{f}_{h}\right|_{1, T} \leq \frac{C}{h_{T}}\left\|\hat{f}_{h}\right\|_{0, T}
$$

Hence, since $\left\|\hat{f}_{h}\right\|_{0, T} \leq C\left\|f_{h}\right\|_{0, \widetilde{T}}$ because of Lemma 2.3 we have

$$
h_{T}^{2}\left\|\hat{f}_{h}-f^{e}\right\|_{1, T} \leq h_{T}^{2}\left(\left\|\hat{f}_{h}\right\|_{1, T}+\left\|f^{e}\right\|_{1, T}\right) \leq C\left(h_{T}\left\|f_{h}\right\|_{0, \widetilde{T}}+h_{T}^{2}\left\|f^{e}\right\|_{1, T}\right) .
$$

Therefore,

$$
\left\|\hat{f}_{h}-f^{e}\right\|_{0, \Omega_{h} \backslash \bar{\Omega}} \leq C\left(\left\|f_{h}-f\right\|_{0, \Omega}+h\left\|f_{h}\right\|_{0, \Omega}+h^{2}\left\|f^{e}\right\|_{1, \Omega_{h}}\right),
$$

and the lemma follows from (2.16), (2.15), and (3.5).

Now we can prove an double order $\mathrm{L}^{2}(\Omega)$-estimate.

Lemma 3.9. There exists a positive constant $C$ such that, for all $f \in \mathrm{H}^{1}(\Omega)$,

$$
\left\|\left(\mathbf{T}-\mathbf{T}_{h}\right) f\right\|_{0, \Omega} \leq C h^{2 r}\|f\|_{1, \Omega} .
$$

Proof. Let $f \in \mathrm{H}^{1}(\Omega)$. Since

$$
\left\|\left(\mathbf{T}-\mathbf{T}_{h}\right) f\right\|_{0, \Omega}=\sup _{g \in \mathrm{L}^{2}(\Omega)} \frac{b\left(\left(\mathbf{T}-\mathbf{T}_{h}\right) f, g\right)}{\|g\|_{0, \Omega}},
$$

then it only remains to prove that

$$
\left|b\left(\left(\mathbf{T}-\mathbf{T}_{h}\right) f, g\right)\right| \leq C h^{2 r}\|f\|_{1, \Omega}\|g\|_{0, \Omega} \quad \forall g \in \mathrm{L}^{2}(\Omega) .
$$

Let $g \in \mathrm{L}^{2}(\Omega)$ and $v=\mathbf{T} g$. We denote $v_{h}=\overline{\mathbf{T}}_{h} \hat{\mathbf{E}} \mathbf{P} g$ and $\check{v}_{h}=\check{\mathbf{E}} v_{h}=\mathbf{T}_{h} g$ as above. Since according to (2.9) $v \in \mathrm{H}^{1+r}(\Omega)$, there exists a bounded extension $v^{e}$ of $v$ satisfying (3.1) for $s=1+r$.

Since $a$ and $b$ are symmetric and $u-\check{u}_{h}=\left(\mathbf{T}-\mathbf{T}_{h}\right) f \in \mathrm{H}^{1}(\Omega)$, then we have

$$
b\left(\left(\mathbf{T}-\mathbf{T}_{h}\right) f, g\right)=a\left(u-\check{u}_{h}, v\right)=a\left(u-\check{u}_{h}, v-\check{v}_{h}\right)+a\left(u-\check{u}_{h}, \check{v}_{h}\right) .
$$

Thus, from the continuity of $a(\cdot, \cdot)$ and Lemma 3.6, we only have to estimate the second term in the right-hand side. Since $\check{v}_{h} \in \mathcal{L}_{h}(\Omega) \subset \mathrm{H}^{1}(\Omega)$ and $v_{h} \in \mathcal{L}_{h}\left(\Omega_{h}\right)$, then

$$
\begin{aligned}
a\left(u-\check{u}_{h}, \check{v}_{h}\right) & =b\left(f, \check{v}_{h}\right)-a\left(\check{u}_{h}, \check{v}_{h}\right) \\
& =\left[b\left(f, \check{v}_{h}\right)-b_{h}\left(\hat{f}_{h}, v_{h}\right)\right]+\left[a_{h}\left(u_{h}, v_{h}\right)-a\left(\check{u}_{h}, \check{v}_{h}\right)\right] .
\end{aligned}
$$

By using (2.14) and repeating the arguments in the proof of Lemma 3.3, we obtain

$$
\begin{aligned}
a\left(u-\check{u}_{h}, \check{v}_{h}\right)= & \int_{\Omega \backslash \bar{\Omega}_{h}} f_{h} \check{v}_{h} d x-\int_{\Omega_{h} \backslash \bar{\Omega}} \hat{f}_{h} v_{h} d x \\
& +\int_{\Omega_{h} \backslash \bar{\Omega}}\left(\nabla u_{h} \cdot \nabla v_{h}+u_{h} v_{h}\right) d x-\int_{\Omega \backslash \bar{\Omega}_{h}}\left(\nabla \check{u}_{h} \cdot \nabla \check{v}_{h}+\check{u}_{h} \check{v}_{h}\right) d x .
\end{aligned}
$$


Thus, to conclude the lemma, we estimate each term in the right-hand side above. From Lemma 2.4 applied to $\check{v}_{h}$ and $f_{h},(2.16)$, and (2.17),

$$
\begin{aligned}
\left|\int_{\Omega \backslash \bar{\Omega}_{h}} f_{h} \check{v}_{h} d x\right| & \leq\left\|f_{h}\right\|_{0, \Omega \backslash \bar{\Omega}_{h}}\left\|\check{v}_{h}\right\|_{0, \Omega \backslash \bar{\Omega}_{h}} \\
& \leq\left(\left\|f_{h}-f\right\|_{0, \Omega \backslash \bar{\Omega}_{h}}+\|f\|_{0, \Omega \backslash \bar{\Omega}_{h}}\right) C h\left\|\check{v}_{h}\right\|_{1, \Omega} \\
& \leq C h^{2}\|f\|_{1, \Omega}\|g\|_{0, \Omega} .
\end{aligned}
$$

By using Lemma 2.4 applied to $v_{h}$ and $f^{e}$, Lemma 3.8, (2.12), (3.5), and (2.17),

$$
\begin{aligned}
\left|\int_{\Omega_{h} \backslash \bar{\Omega}} \hat{f}_{h} v_{h} d x\right| & \leq\left\|\hat{f}_{h}\right\|_{0, \Omega_{h} \backslash \bar{\Omega}}\left\|v_{h}\right\|_{0, \Omega_{h} \backslash \bar{\Omega}} \\
& \leq\left(\left\|\hat{f}_{h}-f^{e}\right\|_{0, \Omega_{h} \backslash \bar{\Omega}}+\left\|f^{e}\right\|_{0, \Omega_{h} \backslash \bar{\Omega}}\right) C h\left\|v_{h}\right\|_{1, \Omega_{h}} \\
& \leq C h^{2}\left(\|f\|_{1, \Omega}+\left\|f^{e}\right\|_{1, \Omega_{h}}\right)\left\|\check{v}_{h}\right\|_{1, \Omega_{h}} \\
& \leq C h^{2}\|f\|_{1, \Omega}\|g\|_{0, \Omega} .
\end{aligned}
$$

By using Remark 3.4, Lemma 2.4, (3.2), and (2.9),

$$
\begin{aligned}
\mid \int_{\Omega_{h} \backslash \bar{\Omega}}(\nabla & \left.u_{h} \cdot \nabla v_{h}+u_{h} v_{h}\right) d x \mid \leq\left\|u_{h}\right\|_{1, \Omega_{h} \backslash \bar{\Omega}}\left\|v_{h}\right\|_{1, \Omega_{h} \backslash \bar{\Omega}} \\
& \leq\left(\left\|u_{h}-u^{e}\right\|_{1, \Omega_{h} \backslash \bar{\Omega}}+\left\|u^{e}\right\|_{1, \Omega_{h} \backslash \bar{\Omega}}\right)\left(\left\|v_{h}-v^{e}\right\|_{1, \Omega_{h} \backslash \bar{\Omega}}+\left\|v^{e}\right\|_{1, \Omega_{h} \backslash \bar{\Omega}}\right) \\
& \leq\left(C h^{r}\|f\|_{0, \Omega}+C h^{r}\left\|u^{e}\right\|_{1+r, \Omega_{h}}\right)\left(C h^{r}\|g\|_{0, \Omega}+C h^{r}\left\|v^{e}\right\|_{1+r, \Omega_{h}}\right) \\
& \leq C h^{2 r}\|f\|_{0, \Omega}\|g\|_{0, \Omega} .
\end{aligned}
$$

Finally, from Lemmas 3.6 and 2.4 and (2.9),

$$
\begin{aligned}
\mid \int_{\Omega \backslash \bar{\Omega}_{h}}\left(\nabla \check{u}_{h} \cdot\right. & \left.\nabla \check{v}_{h}+\check{u}_{h} \check{v}_{h}\right) d x \mid \leq\left\|\check{u}_{h}\right\|_{1, \Omega \backslash \bar{\Omega}_{h}}\left\|\check{v}_{h}\right\|_{1, \Omega \backslash \bar{\Omega}_{h}} \\
& \leq\left(\left\|\check{u}_{h}-u\right\|_{1, \Omega \backslash \bar{\Omega}_{h}}+\|u\|_{1, \Omega \backslash \bar{\Omega}_{h}}\right)\left(\left\|\check{v}_{h}-v\right\|_{1, \Omega \backslash \bar{\Omega}_{h}}+\|v\|_{1, \Omega \backslash \bar{\Omega}_{h}}\right) \\
& \leq\left(C h^{r}\|f\|_{0, \Omega}+C h^{r}\|u\|_{1+r, \Omega}\right)\left(C h^{r}\|g\|_{0, \Omega}+C h^{r}\|v\|_{1+r, \Omega}\right) \\
& \leq C h^{2 r}\|f\|_{0, \Omega}\|g\|_{0, \Omega} .
\end{aligned}
$$

As a consequence of this lemma we may prove a double order error estimate for the eigenfunctions in $\mathrm{L}^{2}$ norm.

Theorem 3.10. Let $\mu_{k}$ be the $k$-th (simple) eigenvalue of $\mathbf{T}$ and $\mu_{h k}$ the $k$-th eigenvalue of $\mathbf{T}_{h}$. Then, the corresponding eigenfunctions $u_{k}$ and $\check{u}_{h k}$ can be chosen such that $\left\|u_{k}\right\|_{0, \Omega}=\left\|\check{u}_{h k}\right\|_{0, \Omega}=1$ and

$$
\left\|u_{k}-\check{u}_{h k}\right\|_{0, \Omega} \leq C h^{2 r}
$$

with $C$ a strictly positive constant.

Proof. Because of the previous lemma and the estimate (2.10), the theorem is also a direct consequence of Lemma 7.1 in [1].

Finally, a double order of convergence for the eigenvalues can also be proved: 
Theorem 3.11. Let $\mu_{k}$ be the $k$-th (simple) eigenvalue of $\mathbf{T}$ and $\mu_{h k}$ the $k$-th eigenvalue of $\mathbf{T}_{h}$. Then, there exists a strictly positive constant $C$ such that

$$
\left|\mu_{k}-\mu_{h k}\right| \leq C h^{2 r} .
$$

Proof. Since $\mathbf{T}$ is self-adjoint with respect to $b(\cdot, \cdot)$, then Theorem 7.3 of [1] applied to our case yields

$$
\left|\mu-\mu_{h}\right| \leq C\left[\sup _{f, g \in \mathcal{E} \subset L^{2}(\Omega)} \frac{\left|b\left(\left(\mathbf{T}-\mathbf{T}_{h}\right) f, g\right)\right|}{\|f\|_{0, \Omega}\|g\|_{0, \Omega}}+\left\|\left.\left(\mathbf{T}-\mathbf{T}_{h}\right)\right|_{\mathcal{E}}\right\|_{0, \Omega}\left\|\left.\left(\mathbf{T}-\mathbf{T}_{h}^{*}\right)\right|_{\mathcal{E}}\right\|_{0, \Omega}\right],
$$

where $\mathcal{E}$ is the eigenspace associated to $\mu_{k}$, and $\mathbf{T}_{h}^{*}$ is the adjoint operator of $\mathbf{T}_{h}$ with respect to $b(\cdot, \cdot)$.

Now

$$
\sup _{f, g \in \mathcal{E} \subset L^{2}(\Omega)} \frac{\left|b\left(\left(\mathbf{T}-\mathbf{T}_{h}\right) f, g\right)\right|}{\|f\|_{0, \Omega}\|g\|_{0, \Omega}}=\sup _{f \in \mathcal{E}} \frac{\left\|\left(\mathbf{T}-\mathbf{T}_{h}\right) f\right\|_{0, \Omega}}{\|f\|_{0, \Omega}}
$$

and

$$
\begin{aligned}
\left\|\left(\mathbf{T}-\mathbf{T}_{h}^{*}\right) f\right\|_{0, \Omega} & =\sup _{g \in \mathrm{L}^{2}(\Omega)} \frac{b\left(\left(\mathbf{T}-\mathbf{T}_{h}^{*}\right) f, g\right)}{\|g\|_{0, \Omega}}=\sup _{g \in \mathrm{L}^{2}(\Omega)} \frac{b\left(f,\left(\mathbf{T}-\mathbf{T}_{h}\right) g\right)}{\|g\|_{0, \Omega}} \\
& \leq\|f\|_{0, \Omega} \sup _{g \in \mathrm{L}^{2}(\Omega)} \frac{\left\|\left(\mathbf{T}-\mathbf{T}_{h}\right) g\right\|_{0, \Omega}}{\|g\|_{0, \Omega}} \leq C\|f\|_{0, \Omega},
\end{aligned}
$$

the last inequality because of (2.8) and (2.17).

Thus, the theorem is a consequence of Lemma 3.9 and estimate (2.10).

\section{Conclusions}

We have analyzed the finite element approximation of eigenvalues and eigenfunctions of the Laplace equations with Neumann boundary conditions on curved (nonconvex) domains $\Omega$. Convergence and optimal order error estimates have been proved for standard piecewise linear elements on a discrete polygonal domain $\Omega_{h} \not \subset \Omega$, in the framework of the abstract spectral approximation theory as stated in [1]. Similar results had been previously proved by Vanmaele and Ženíšek [19] (based on min-max techniques), and Lebaud [14], but in both cases only for Dirichlet boundary conditions.

\section{REFERENCES}

1. I. Babuška and J. Osborn, Eigenvalue problems in Handbook of Numerical Analysis, Vol II, P.G. Ciarlet and J.L. Lions, eds., North Holland, Amsterdam, 1991, pp. 641-787. CMP 91:14

2. C. Bernardi, Optimal finite-element interpolation on curved domain, SIAM J. Numer. Anal., 5 (1989) 1212-1240. MR 91a:65228

3. D. Boffi, F. Brezzi, and L. Gastaldi, On the problem of spurious eigenvalues in the approximation of linear elliptic problems in mixed form, Math. Comp., 69 (2000) 121-140. MR 2000i:65175

4. D. Boffi, F. Brezzi, and L. Gastaldi, On the convergence of eigenvalues for mixed formulations, Ann. Scuola Norm. Sup. Pisa Cl. Sci. (4), 25 (1998) 131-154. MR 99i:65121

5. S. Brenner and L.R. Scott, The mathematical theory of finite element methods, SpringerVerlag, New York, 1994. MR 95f:65001

6. J.H. Bramble and J.T. King, A robust finite element method for nonhomogeneous Dirichlet problems in domains with curved boundaries, Math. Comp., 63 (1994) 1-17. MR 94i:65112

7. P.G. Ciarlet, Basic error estimates for elliptic problems, in Handbook of Numerical Analysis, Vol. II, P.G. Ciarlet and J.L. Lions, eds., North Holland, Amsterdam, 1991, pp. 17-351. MR 92f:65001 
8. P.G. Ciarlet and P.A. Raviart, Interpolation theory over curved elements with applications to finite element methods, Comput. Methods Appl. Mech. Engrg., 1 (1972) 217-249. MR 51:11991

9. T. Dupont and R. Scott, Polynomial approximation of functions in Sobolev spaces, Math. Comp., 34 (1980) 441-463. MR 81h:65014

10. M. Feistauer, On the finite element approximation of functions with noninteger derivatives Numer. Funct. Anal. and Optim., 10 (1989) 91-110. MR 90b:65009

11. M. Feistauer and A. Ženíšek, Finite element solution of nonlinear elliptic problems, Numer. Math., 50 (1987) 451-475. MR 88f:65195

12. V. Girault and P.A. Raviart, Finite element methods for Navier-Stokes equations, SpringerVerlag, Germany, Berlin, 1986. MR 88b:65129

13. P. Grisvard, Elliptic Problems On Nonsmooth Domains, Pitman, Boston, London, Melbourne, 1985. MR 86m:35044

14. M.P. Lebaud, Error estimate in an isoparametric finite element eigenvalue problem, Math. Comp., 63 (1994) 17-40. MR 95e:65102

15. J. Osborn, Spectral approximation for compact operators, Math. Comp., 29 (1965) 712-725. MR 52:3998

16. P.A. Raviart and J.M. Thomas, Introduction à l'Analyse Numérique des Equations aux Dérivées Partielles, Masson, Paris, 1983. MR 87a:65001a

17. R. Scott, Interpolated boundary conditions in the finite element method, SIAM J. Numer. Anal., 12 (1975) 440-427. MR 52:7162

18. G. Strang and G. Fix, An Analysis of the Finite Element Method, Prentice-Hall, Englewood Cliffs, 1973. MR 56:1747

19. M. Vanmaele and A. Ženíšek, External finite element approximations of eigenvalue problems, $\mathrm{M}^{2} \mathrm{AN}, 27$ (1993) 565-589. MR 94j:65135

20. M. Vanmaele and A. Ženíšek, External finite-element approximations of eigenfunctions in the case of multiple eigenvalues, J. Comput. Appl. Math., 50 (1994) 51-66. MR 95f:65199

21. M. Vanmaele and A. Ženíšek, The combined effect of numerical integration and approximation of the boundary en the finite element methods for the eigenvalue problems, Numer. Math., 71 (1995) 253-273. MR 96j:65112

22. A. Ženíšek, Nonlinear Elliptic and Evolution Problems and Their Finite Element Approximation, Academic Press, London, 1990. MR 92c:65003

23. M. Zlámal, Curved elements in the finite element method I, SIAM J. Numer. Anal., 10 (1973) 228-240. MR 52:16060

Departamento de Ingeniería Matemática, Universidad de Concepción, Casilla 160-C, CONCEPCión, Chile

E-mail address: erwin@ing-mat.udec.cl

Departamento de Ingeniería Matemática, Universidad de Concepción, Casilla 160-C, Concepción, Chile

E-mail address: rodolfo@ing-mat.udec.cl 